\title{
Fast Kicker Requirements for the SSC's Low and Medium Energy Boosters
}

\author{
M. Wilson, L. Schneider, D. Anderson, C. Pappas \\ Superconducting Supercollider Laboratory ${ }^{\dagger}$ \\ Accelerator Division \\ 2550 Beckleymeade Avenue \\ Dallas, Texas 75237
}

\section{Abstract}

Requirements for the transfer kickers that move beam from the Low-Energy Booster (LEB) to the Medium-Energy Booster (MEB) are being established. Magnetic field intensity, pulse shapes, and available space for the kicker magncts within the ring beamlines are known to a level that allows preliminary design to begin on these systems. This fast kicker system, which must transfer the entire $12 \mathrm{GeV} / \mathrm{c}$ LEB batch to the MEB, is required to produce $150-200 \mathrm{G}$ fields that rise in 60 $80 \mathrm{~ns}$, with a pulsewidth of $1.9 \mu \mathrm{s}$. The present MEB filling scenario requires this kicker system to operate in a 6-7 shot burst mode at repetition rates as fast as $10 \mathrm{~Hz}$.

\section{REQUIREMENTS}

The $570 \mathrm{~m}$ LEB, when completely filled, holds 114 proton bunches spaced $5.0 \mathrm{~m}$ apart ( $21 \mathrm{~ns}$ at the injection energy of $600 \mathrm{MeV} / \mathrm{c}$ ). After acceleration to the top-end energy of 12 $\mathrm{GeV} / \mathrm{c}$, the revolution period is reduced to approximately 1.9 $\mu \mathrm{s}$ ( $16.7 \mathrm{~ns} / \mathrm{bunch}$ spacing). One filling scenario for the collider, shown in Figure 1, requires that the MEB be filled with six LEB batches, each containing 109 proton bunches, with a three-bunch gap between LEB batches. Three such MEB fills, with 25-bunch (400ns) gaps between, completely fill the High Energy Booster (HEB) while leaving an appropriate abort gap of $1.7 \mu \mathrm{s}$ (103 bunches). Eight full HEB cycles would then fill each collider ring.

Filling the MEB with 109-bunch trains implies that five of the original bunches must be removed during the transfer. In order to reduce the radiation levels at the MEB injection point and not overly constrain the MEB injection kicker risetime, four bunches are lost at the LEB ejection point and the fifth is lost at the MEB injection point. This allows the risetime of the LEB ejection kicker magnet to be up to $80 \mathrm{~ns}$ (five $16.7 \mathrm{~ns}$ bunch spacings, minus one bunch width of $3.3 \mathrm{~ns}$ ). Packing the LEB batches in the MEB requires the risetime of the MEB injection kicker to be less than 63 ns (four bunch spacings minus one bunch width). The pulsewidth of both the LEB ejection and the MEB injection kickers must be at least the full LEB revolution time of $1.9 \mu \mathrm{s}$. To control overall beam emittance growth, the magnetic field amplitude must vary over the entire pulsewidth by less than \pm 1.0 percent, and shot-toshot variations must also be kept to this level.

The vertical ejection scheme for the LEB includes a fourmagnet local orbit bump, a kicker magnet system, and two ejection septum magnets. In order to produce the required downstream deflection, the ejection kicker magnet must produce an integrated field of 600-750 G-m. Approximately $6.5 \mathrm{~m}$ of space has been allocated in the LEB ring for the kicker magnets. The internal dimensions of the vacuum chamber at the kicker magnet location are dictated by the size of the 600 $\mathrm{MeV} / \mathrm{c}$ injected beam, and has been set at $6.5 \mathrm{~cm}$ wide by 8.0 $\mathrm{cm}$ high. The 'good-field' region, defined as the area in which the magnetic field varies by less than 0.5 percent, must be 2.0 $\mathrm{cm}$ wide by $5.0 \mathrm{~cm}$ high.

For MEB injection, the required total magnetic field is approximately $480 \mathrm{G}-\mathrm{m}$, with at least $6.0 \mathrm{~m}$ available for kicker magnet hardware in the beamline. The magnet aperture is presently set at $10.0 \mathrm{~cm}$ wide by $5.0 \mathrm{~cm}$ high, with the 'good-field' region approximately the same as that in the LEB ejection kicker. Injection into the MEB is in the horizontal direction.

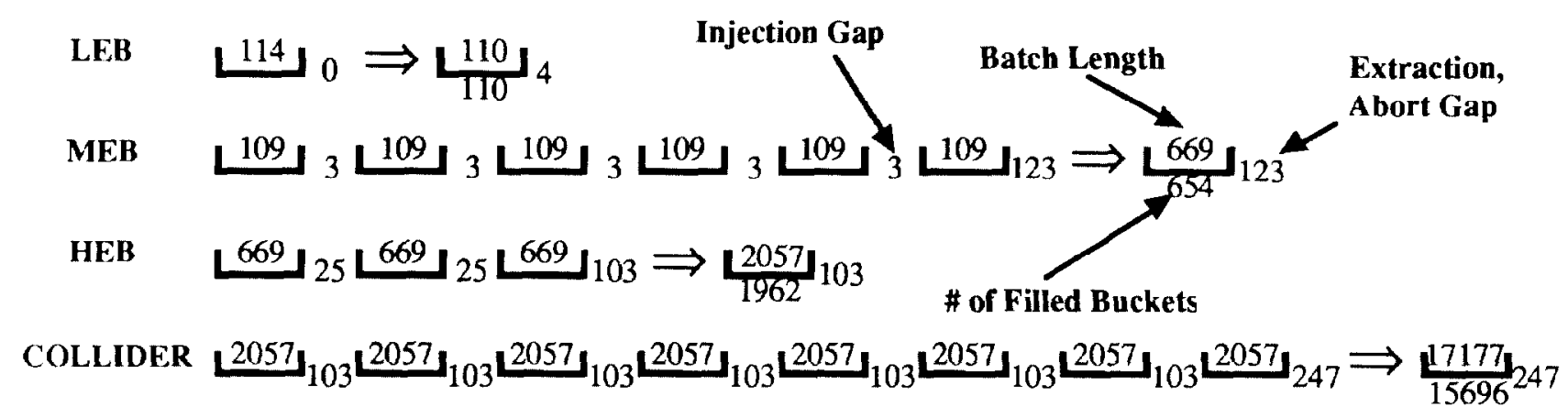

Figure 1. Collider Ring Filling Scenario. Batch Lengths and Gaps are Represented in Number of Beam Buckets, with Each Bucket Equal to $5.0 \mathrm{~m}$ of Ring Circumference, or $16.68 \mathrm{~ns}$ in Time at Relativistic Energies.

${ }^{\dagger}$ Operated by the Universities Research Association, Inc., for the U. S. Department of Energy under Contract No. DEAC02-89ER40486.

U.S. Government work not protected by U.S. Copyright. 


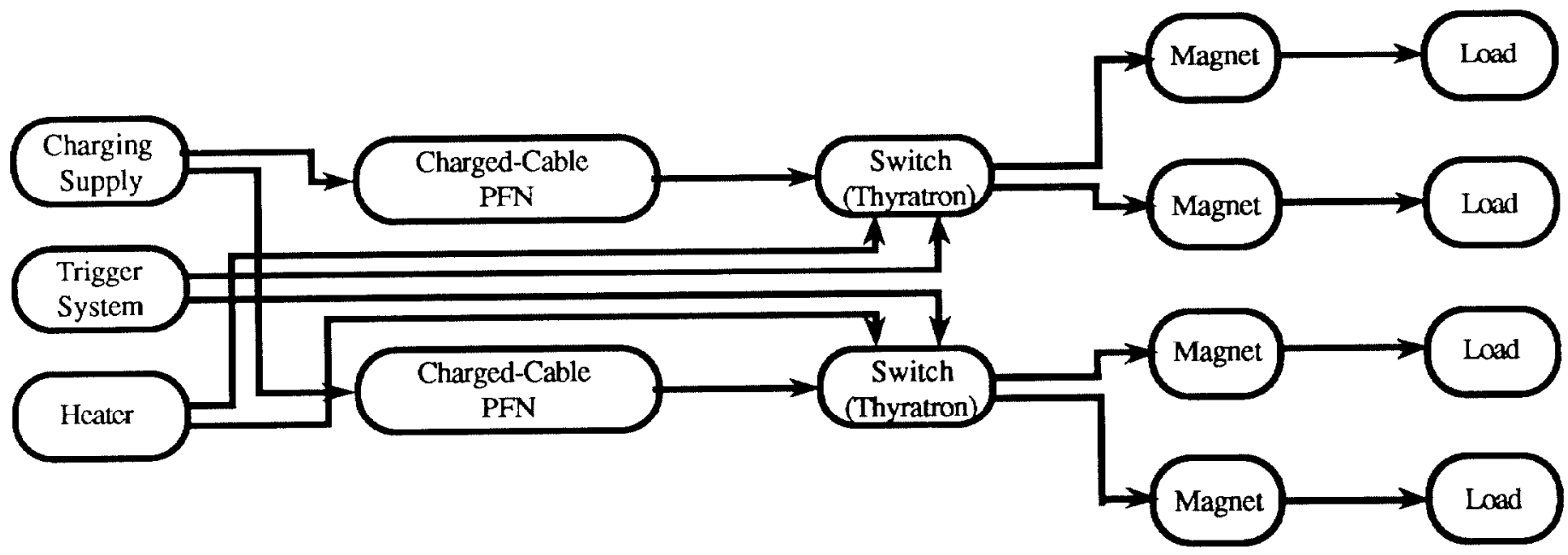

Figure 2. Block Diagram of Kicker Systems

\section{LEB EJECTION KICKER SYSTEM DESIGN}

All of the SSC ejection and injection kicker systems consist of, as a minimum, (a) a charging system, (b) a triggering system, (c) a pulse-forming network (PFN), (d) a triggered switch, (e) a pulsed magnet, and (f) a terminating load. Such a system is shown in Figure 2. The short pulsewidth of the LEB ejection kicker allows the use of charged cable as the pulseforming network. Standard RG-220 $50 \Omega$ cable is the preliminary choice, as it has proven reliability in kickers and other high-voltage pulsed and DC systems[1]. The output voltage pulse from the charged cable is half the charge voltage and twice the electrical length of the cable. For the LEB ejection and MEB injection kickers, 190 $\mathrm{m}$ cables will be required. Other system parameters are driven by the magnet requirements presented above.

The available length for the LEB ejection kickers cannot be filled entirely with magnets. Terminations, flanges, bellows, and vacuum hardware combine to limit the total space for actual magnetic material to $4.8 \mathrm{~m}$. This implies a maximum field magnitude over the length of the magnet of $156 \mathrm{G}$.

Figure 3 is a cross-sectional view of a possible LEB ejection kicker magnet. The magnet aperture (gap $x$ pole width) is 7.5 by $9.0 \mathrm{~cm}$, which includes a one centimeter allowance in each dimension for the beampipe wall and tolerance build-up. For a C-magnet, with $\mu_{\mathrm{r}}=40, w=9 \mathrm{~cm}$, $\mathrm{g}=7.5 \mathrm{~cm}, \mathrm{c}$ (the ferrite pathlength) $=30 \mathrm{~cm}$, and $\mathrm{t}$ (ferrite thickness $)=4 \mathrm{~cm}$, the inductance is

$$
\mathrm{L}=\mu_{0} w /\left(\mathrm{g}+\mathrm{wc} / \mathrm{t}_{\mathrm{r}}\right)=1.23 \mu \mathrm{h} / \mathrm{m},
$$

and the current necessary to deliver the required B is

$$
\mathrm{I}=\mathrm{B}\left(\mathrm{g}+\mathrm{wc} / \mathrm{\mu}_{\mathrm{T}}\right) / \mu_{0}=1140 \mathrm{~A} .
$$

Assuming the maximum voltage that can be delivered is 30 $\mathrm{kV}$ (implying maximum charge and switch voltages of 60 $\mathrm{kV}$ ), the maximum modulator source impedance is $26 \Omega$. Two parallel RG-220 cables, with an equivalent impedance of $2.5 \Omega$, results in the electrical length of the magnet equaling

$$
v^{-1}=\sqrt{ } L C=L / Z_{0}=1.23 \mu \mathrm{h} / 25 \Omega=50 \mathrm{~ns} / \mathrm{m} .
$$

To meet the risetime requirements, it is expected that the magnet lengths will be less than $0.5 \mathrm{~m}$, so that no more than 25 ns of the cquivalent magnetic field risetime is due to the transit time of the magnet.

In order to match the magnet to the input cable impedance, the capacitance of the busbar to ground must be

$$
\mathrm{C}=\mathrm{L} / \mathrm{Z}_{\mathrm{o}}{ }^{2}=2.0 \mathrm{nF} / \mathrm{m}
$$

The capacitance of the busbar to the outer case is approximately $0.2 \mathrm{nF} / \mathrm{m}$, so additional capacitance must be added. As is shown in Figure 3, this might be accomplished by using the ceramic beampipe as a stripline capacitor, with the inside of the pipe nearest the busbar coated with conductor and grounded at one end. If $3 \mathrm{~mm}$ thick busbars are used, this conducting strip must be approximately $6 \mathrm{~cm}$ wide, which fits well within the $6.5 \mathrm{~cm}$ inner dimension of the beampipe. The remaining inside walls of the beampipe will be coated with high-resistivity material to provide a path for static charge bleed-off, while allowing fast magnetic field penetration.

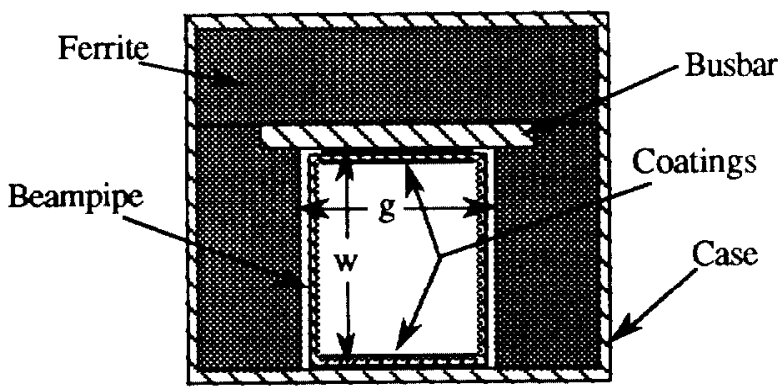

Figure 3. Cross-Section of Possible LEB Injection Kicker Magnet.

Thyratrons are expected to be the switch choice for the fast kicker systems. Assuming four cables are switched by each thyratron (at a voltage of $60 \mathrm{kV}$ ) and the current risetime is approximately $30 \mathrm{~ns}$, the switch $\mathrm{dI} / \mathrm{dt}$ is $4.6 \mathrm{kA} / 30 \mathrm{~ns}=1.5 \mathrm{x}$ $10^{11} \mathrm{~A} / \mathrm{s}$. This value is within the range of existing thyratrons such as the EEV CX1725.[2] 


\section{MEB INJECTION KICKER SYSTEM DESIGN}

Figure 4 depicts a possible cross-section for the MEB injection kicker magnets. Using the above formulas, with a polewidth of $11 \mathrm{~cm}$ and a gap of $6 \mathrm{~cm}$, the inductance of the busbar will be $1.72 \mu \mathrm{h} / \mathrm{m}$. Assuming that 4.8 of the available $6.0 \mathrm{~m}$ will be magnetic material implies a magnetic field within the magnet of $100 \mathrm{G}$. The current required to generate this magnetic field is approximately $640 \mathrm{~A}$. Selecting $50 \Omega$ as the impedance for the busbar results in cable and switch voltage of $64 \mathrm{kV}$ and busbar voltage of $32 \mathrm{kV}$. The capacitance of the busbar to ground must be $690 \mathrm{pF} / \mathrm{m}$ to match the $50 \Omega$ input impedance. Approximately one-quarter of this capacitance exists between the busbar and the grounded magnet case; the remainder can be easily obtained using the conductive coating design described above.

The inverse velocity of the magnet will be $1720 \mathrm{nh} / 50 \Omega=$ $34 \mathrm{~ns} / \mathrm{m}$. By splitting the $4.8 \mathrm{~m}$ magnet length into eight 0.6 $\mathrm{m}$ magnets, $20 \mathrm{~ns}$ transit times are obtained. Two modulators, each consisting of a single thyratron and four RG-220 cables, are required to drive the entire system of eight magnets. The total current of $2.6 \mathrm{kA}$ is required to rise in approximately 20 $\mathrm{ns}$, for an average $\mathrm{dI} / \mathrm{dt}$ of $1.3 \times 10^{11} \mathrm{~A} / \mathrm{s}$. This current rate of rise is also within the range of existing thyratrons.

\section{SUMMARY}

Requirements for the LEB-to-MEB transfer kicker systems are determined, in large part, by the chosen collider filling scenario. For the presented filling scheme, the LEB ejection kickers must gencrate 100-150 G magnetic ficlds in less than $80 \mathrm{~ns}$ with pulse flatness of better than 1.0 percent over the pulsewidth of $1.9 \mu \mathrm{s}$. The system to generate the required

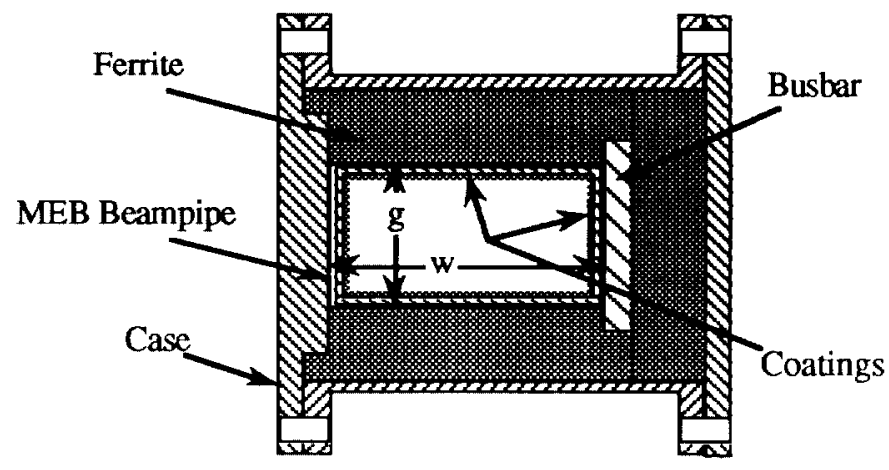

Figure 4. Cross-Section of Possible MEB Injection Kicker Magnet

fields is envisioned as a set of eight or more ferrite magnets, each less than $0.5 \mathrm{~m}$ in length, driven by at least four thyratron-switched charged cable modulators. The MEB injection kickers must generate approximately $100 \mathrm{G}$ fields in less than $65 \mathrm{~ns}$ with similar pulse flatness and shot-to-shot repeatibility. This system has been scoped as a set of eight $0.6 \mathrm{~m}$ magnets driven by two modulators that are identical to those used to drive the LEB ejection system.

\section{REFERENCES}

[1] C. Crawford, J. Dinkel, J. Lackey private communication, February, 1991.

[2] H. Menown, C. A. Pirrie, "Advanced Thyratrons as Switches for the Nineties," 17th Mower Mod. Symp, 86CH2262-4, Seattle, WA, 1986. 